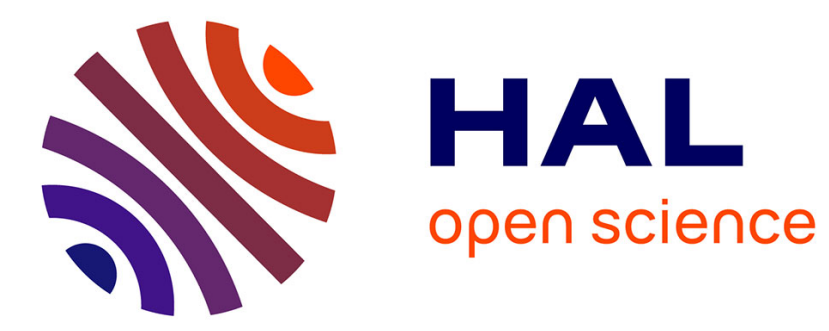

\title{
Stable spins in the zero temperature spinodal decomposition of 2D Potts models
}

\author{
Bernard Derrida, P.M.C. de Oliveira, D. Stauffer
}

\section{To cite this version:}

Bernard Derrida, P.M.C. de Oliveira, D. Stauffer. Stable spins in the zero temperature spinodal decomposition of 2D Potts models. Physica A: Statistical Mechanics and its Applications, 1996, 224 (3-4), pp.604-612. 10.1016/0378-4371(95)00345-2 . hal-03285606

\section{HAL Id: hal-03285606 https://hal.science/hal-03285606}

Submitted on 21 Jul 2021

HAL is a multi-disciplinary open access archive for the deposit and dissemination of scientific research documents, whether they are published or not. The documents may come from teaching and research institutions in France or abroad, or from public or private research centers.
L'archive ouverte pluridisciplinaire HAL, est destinée au dépôt et à la diffusion de documents scientifiques de niveau recherche, publiés ou non, émanant des établissements d'enseignement et de recherche français ou étrangers, des laboratoires publics ou privés. 


\title{
Stable spins in the zero temperature spinodal decomposition of 2D Potts models
}

\author{
B. Derrida ${ }^{a}$, P.M.C. de Oliveira ${ }^{\text {b,c }}$, D. Stauffer ${ }^{\text {b,d }}$ \\ "Laboratoire de Physique Statistique, ENS, 24 rue Lhomond, F-7523I Paris cedex 05, France \\ h Laboratoire de Physique et Mécanique des Milieux Hétérogènes, Ecole Supérieure de Physique et Chimie \\ Industrielles, 10 rue Vauquelin, F-75231 Paris cedex 05. France \\ - Permanently at Instituto de Física, Universidade Federal Fluminense, Av. Litorânea s/n, Boa Viagem, \\ Niterói RJ 24210-340, Brazil \\ ¿ Permanently at Institute for Theoretical Physics, Cologne University, D-50923 Köln. Germany
}

Received 23 September 1995; revised 5 October 1995

\begin{abstract}
We present the results of zero temperature Monte Carlo simulations of the $q$-state Potts model on a square lattice with either four or eight neighbors, and for the triangular lattice with six neighbors. In agreement with previous works, we observe that the domain growth process gets blocked for the nearest-neighbor square lattice when $q$ is large enough, whereas for the eight neighbor square lattice and for the triangular lattice no blocking is observed. Our simulations indicate that the number of spins which never flipped from the beginning of the simulation up to time $t$ follows a power law as a function of the energy, even in the case of blocking. The exponent of this power law varies from less than $1 / 2$ for the Ising case $(q=2)$ to 2 for $q \rightarrow \infty$ and seems to be universal. The effect of blocking on this exponent is invisible at least up to $q=7$.
\end{abstract}

PACS: $02.50 ; 05.20$

Spinodal decomposition is the phase separation process if initially one has a random mixture and the temperature is such that in equilibrium two or more phases coexist [1-5]. Small domains form randomly and then grow in a self similar way until the domain size becomes of the order of the system size. This effect can be studied at zero temperature, where the dynamics reduces to that of a system trying to minimize its energy [6-11]. The growth of domains at zero temperature dynamics may be relevant for problems as different as soap froths [12,13], polycrystals [14] or spin glasses $[6,15,16]$. The goal of the present work is to measure the $q$ dependence of the exponents governing the time decay of the number of spins which never flip during the growth process for two-dimensional systems. Recently, these exponents have been calculated exactly for 
the one-dimensional $q$-state Potts model [17] and have been measured in Monte Carlo simulations for the Ising model in higher dimensions [10,9]. For the Ising model, it was found that the fraction $F(t)$ of the spins which have never flipped up to time $t$ decays like a power law

$$
F(t) \sim t^{-\theta}
$$

with $\theta=3 / 8$ in one dimension, $\theta \simeq 0.22$ in two, and smaller values (possibly blocking) in higher dimensions. This power law is valid for intermediate times corresponding to many Monte Carlo steps per site but to domain sizes much smaller than the lattice size. For the one-dimensional Potts model, $\theta$ is known exactly for all $q$ : it varies from 0 to 1 as $q$ varies continuously from 1 to $\infty[17,18]$. Here we repeat the Monte Carlo simulations for the general $q$-state Potts model [19,7,8,20,21] in dimension two.

Monte Carlo simulations of the zero temperature dynamics of the Potts model are very easy to perform. One starts with a configuration where each spin is given a random color between 1 and $q$ and one lets the system evolve according to a zero temperature dynamics. Basically when a spin is updated, it always changes its orientation if this lowers the energy, never changes it if this would increase the energy, and makes a random choice if the energy stays constant. Then one measures the fraction $F(t)$ of the spins which have never flipped up to time $t$.

One difficulty with zero temperature dynamics is that the system cannot overcome finite energy barriers and that the growth process can be completely stopped or at least greatly slowed down by the existence of blocked configurations. Previous simulations on the two-dimensional $q$-state Potts model have shown that for the Potts model on a square lattice with nearest neighbor interactions (NN), such blocking effects do occur when $q$ is large enough $[7,8,20]$. However, no blocking was seen when simulations are done on the square lattice with nearest and next nearest neighbor interactions (NNN) or on the triangular lattice (TR).

In the present work, in order to test universality, we report the results of simulations done on these three two-dimensional lattices (NN, TR, NNN) for $q$ varying from 2 and $\infty$. We used different random number generators and different updating orders (regular or random), but the asymptotic exponents seem to be the same. Also the exponents seem to be the same whether the spin always selects the color dominating among its neighbors, or compares its present energy with that obtained by flipping to a randomly selected color. We used lattice sizes up to $3000 \times 3000$ and up to $10^{5}$ sweeps through the lattice (which measure the time $t$ ). Both one-word-per-spin and multispin coding techniques [23] were used, and in total of the order of $10^{3}$ workstation hours were spent.

Fig. I illustrates the main behavior, for $q=3,7$, and $\infty$, for the three lattices. Only the square lattice with nearest neighbor interactions (NN), for large enough $q$ shows blocking, i.e. a flattening of the decay of $F(t)$ towards zero $\theta$. For $q=3$ no evidence for blocking is seen in the case of the NN square lattice. For the other two lattices (TR and NNN), even high $q$ values showed no indication of blocking, for millions of sites and thousands of Monte Carlo steps. (For $q=4$ on the NN lattice, the effective exponent 

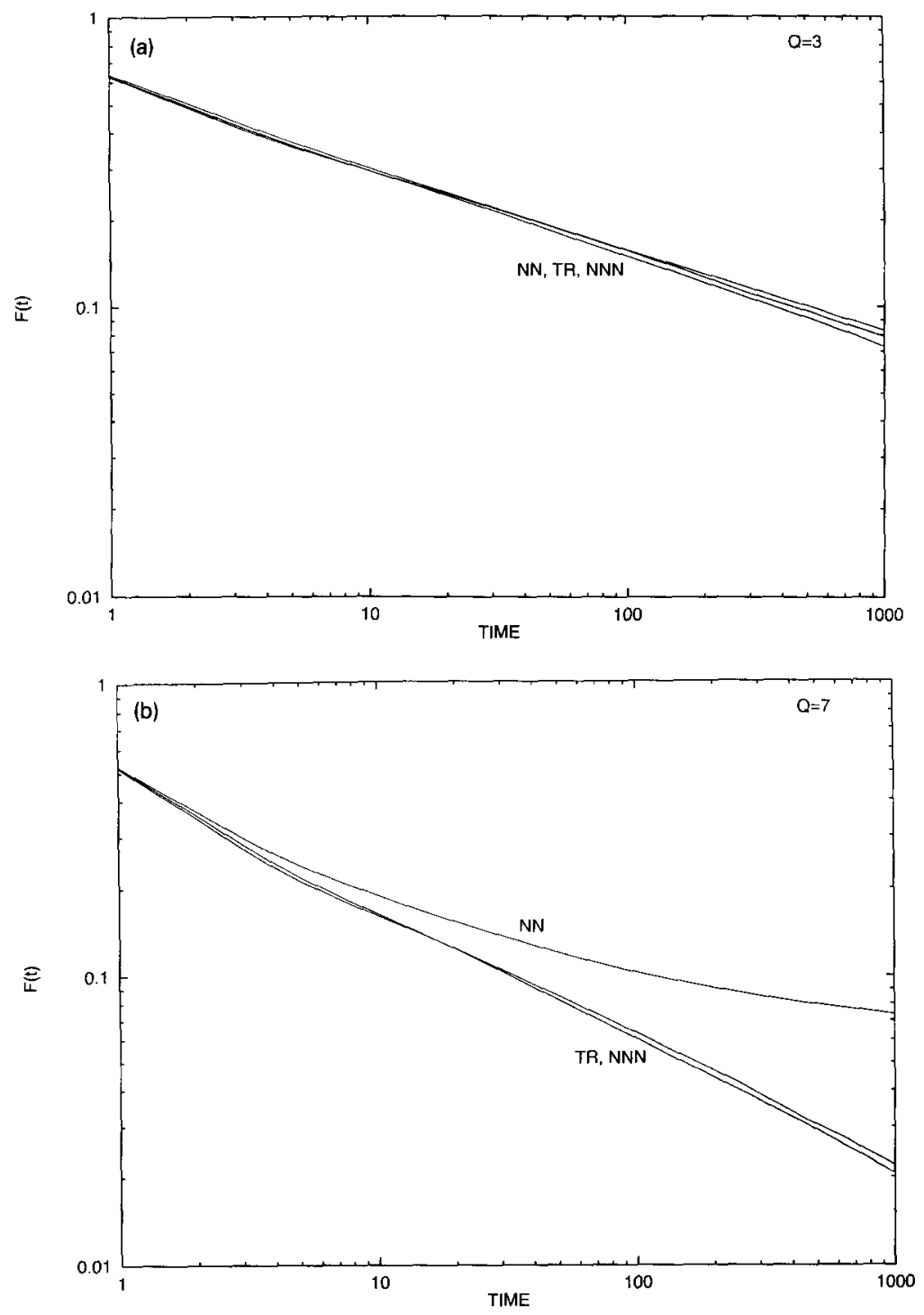

Fig. 1. Frozen fraction versus time for the square lattice with nearest neighbor interactions (NN), the triangular lattice (TR) and the square lattice with nearest and next to nearest neighbor interactions (NNN). The system size is $L=1000$. Parts (a),(b), (c) correspond to $q=3,7, \infty$.

decreased smoothly with increasing time, possibly towards zero, with $t<4 \times 10^{4}$ in a $2000 \times 2000$ lattice; from $q=5$ the blocking looks similar up to $q=\infty$.)

Naively one might think that the three-state Potts model should lead to a rapid blocking of the phase separation, for the square lattice with four neighbors: if the upper part of the lattice has color 1 , and the lower half is vertically divided into the left half with color 2 and the right half with color 3 , then each of the spins has at least two neighbors of its own color, and at most one neighbor for each of the other two colors. 


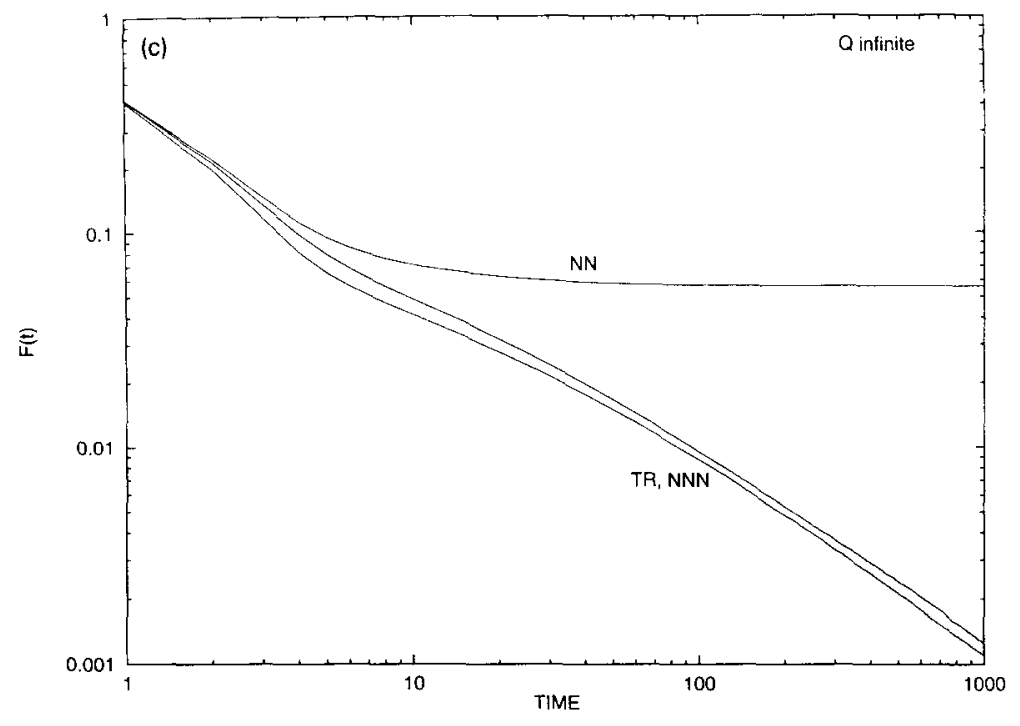

Fig. 1 - continued.

Thus nothing moves at zero temperature. Apparently, such blocking configurations only occur for $q>4$. For $q=3$, they are constantly removed by disturbances coming from far away. This is similar to bootstrap percolation [22] (where an empty square surrounded by a fully occupied plane is completely stable if sites remain occupied only if at least three of their four neighbors on the square lattice are occupied). There too, disturbances from far away remove the blocking, and the apparent blocking concentration for finite lattices vanishes logarithmically if the lattice size goes to infinity.

If $t \rightarrow \infty$ at fixed linear lattice dimension $L$, finite-size effects appear if the domain size approaches the order of $L$. Fig. 2 illustrates that finite-size corrections become visible at about $t \simeq L^{2} / 10$. Fig. 3 shows, however, that for $L=1000$ and more and $t<10^{4}$ such effects can be neglected: the blocking for $q>4$ on the NN lattice does not seem to be a finite-size effect. Of course, we cannot exclude that blocking would occur also in the other cases if we would look at longer times for larger lattices.

The difference between $q<4$ and $q>4$ in the nearest-neighbor square lattice (unblocked versus blocked, respectively), which is not seen for the other two lattices, shows universality for $\theta$ to be invalid. For the other two lattices (TR and NNN), our data lead, nevertheless, to the same estimate of $\theta$ given in Table 1 . However, as $q$ increases, the data, in a log-log plot, show some curvature, indicating that the asymptotic regime has not yet been reached, so that the estimates of $\theta$ given in Table 1 are just effective exponents valid for the range $t<10^{4}$.

An important property of domain growth problems is obviously the time dependence of the linear size of domains $D(t)[7,6,8,20,21]$. Under the assumption that the structure of the domain boundaries is not fractal, this domain size can be related to the energy (per site) $E(t)$ by

$$
D(t) \simeq E^{-1}(t)
$$



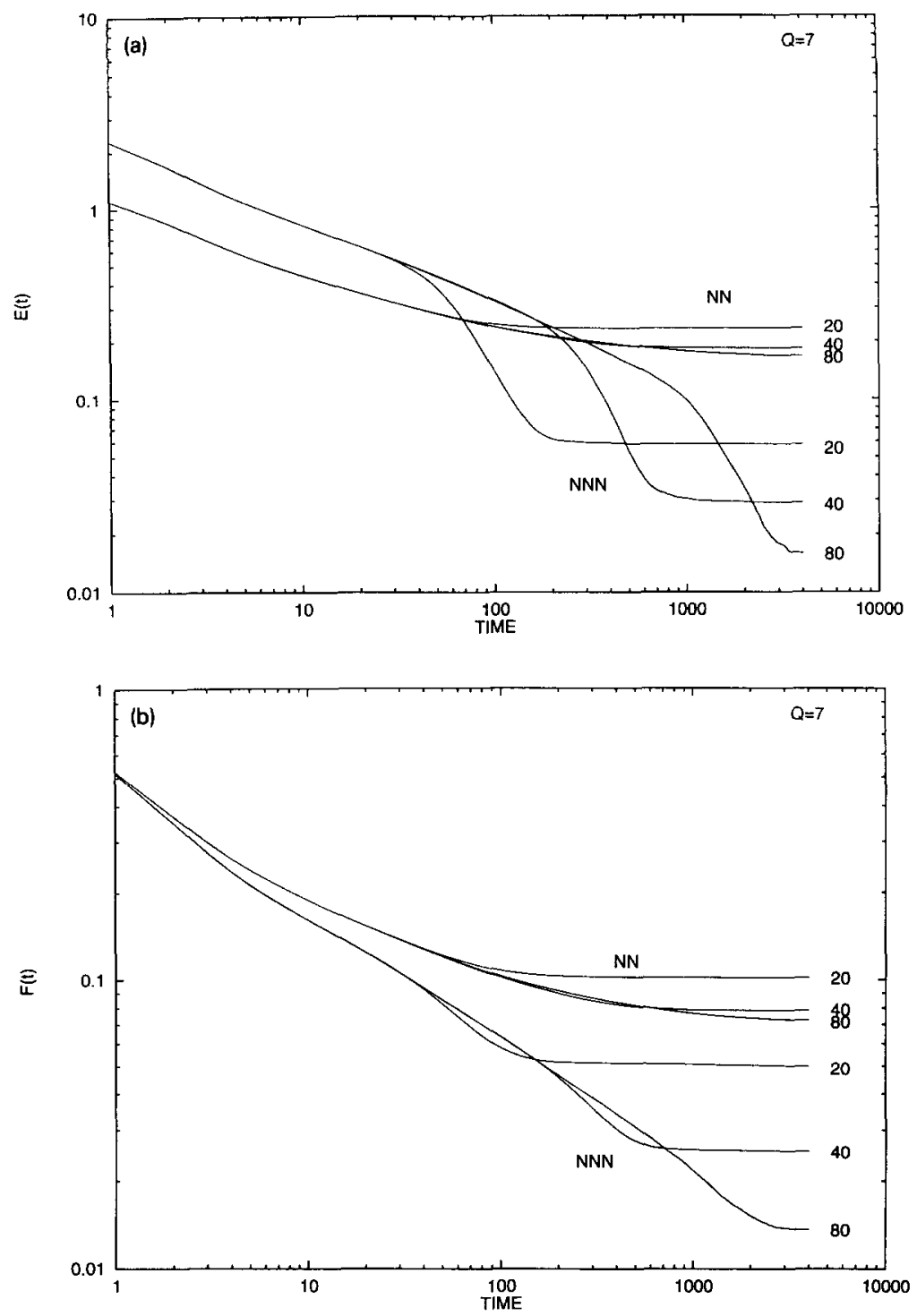

Fig. 2. Finite size effects for $q=7$ and $L=20,40,80$ (averaged over many samples) for the two lattices (NN and NNN).

because at zero temperature there is no contribution to the energy coming from the bulk of the domains [6]. We measured the energy $E(t)$ versus time and found results very similar to those obtained for $F(t)$,

$$
E(t) \sim t^{-\psi}
$$

with blocking effects for the NN square lattice and only effective exponents (due to curvature) for the other two lattices. This is in full agreement with previous works $[8,20]$ who found that the effective exponent $n$ of the domain size $D(t) \sim t^{n}$ is 


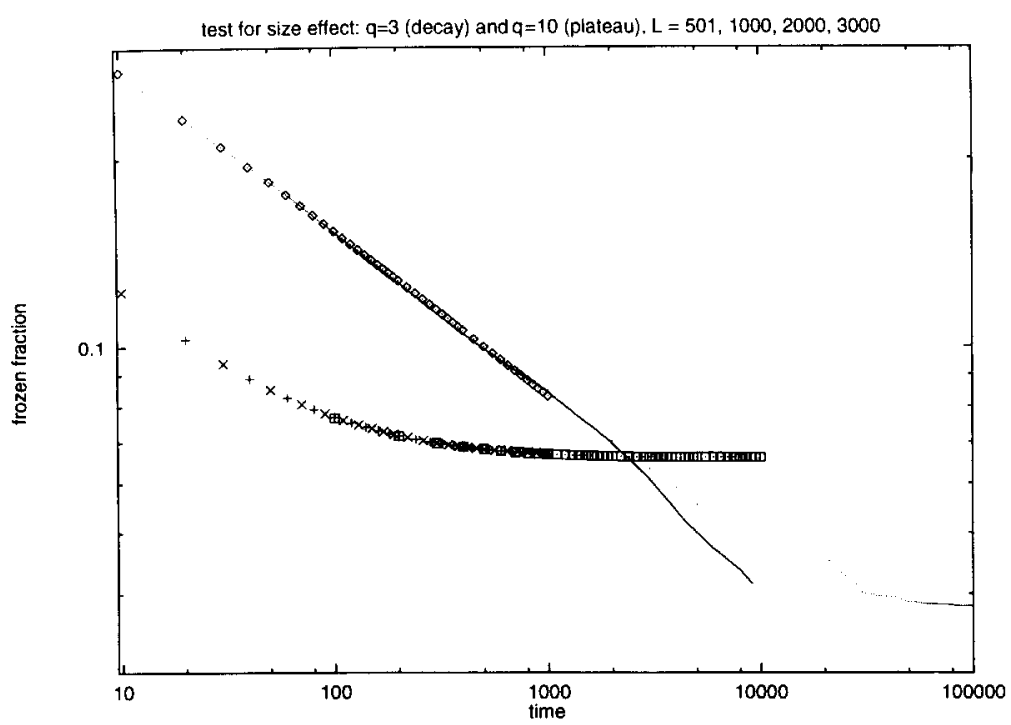

Fig. 3. Comparison of $F(t)$ for $L=1000,2000,3000$ for $\mathrm{NN}$ (the square lattice with nearest neighbor interactions). (Small dots: $L=501$; solid line and squares: $L=1000$; crosses and diamonds: $L=2000$ and 3000.) $q=3$ leads to decay (negative slope) and $q=10$ to blocking (approach towards plateau), both independently of $L$. Only for $L=500$ are size effects visible.

Table 1

The exponents $\theta, \psi$, and $\phi$ as a function of $q$

\begin{tabular}{llll}
\hline$q$ & $\theta$ & $\psi$ & $\phi$ \\
\hline 2 & .21 & .51 & .44 \\
3 & .29 & .48 & .65 \\
4 & $(.36)$ & $(.49)$ & .77 \\
5 & $(.39)$ & $(.45)$ & .86 \\
7 & $(.44)$ & $(.44)$ & 1.01 \\
12 & $(.56)$ & $(.45)$ & 1.17 \\
50 & $(.66)$ & $(.45)$ & 1.57 \\
$\infty$ & $(.86)$ & $(.46)$ & 2.0 \\
\hline
\end{tabular}

The values in parentheses are just effective exponents, as the log-log plots showed some curvature.

less than $1 / 2$ whereas one expects $n=1 / 2$ in the asymptotic regime. We did a long simulation for $q=7$, and this confirmed that the effective exponent $\psi$ increases steadily with time, in full compatibility with $\psi=1 / 2$ asymptotically.

Surprisingly, universality can be partially restored if instead of time $t$ we use energy $E(t)$ as the variable to express a power law for the frozen fraction $F(t)$,

$$
F \sim E^{\phi}
$$

The data show much less curvature than in the case of $\theta$ or of $\psi$ and the exponent $\phi$ measured that way is given in Table 1 . For $q \leq 7$, all the data corresponding to the three lattices give roughly compatible values for $\phi$ whereas for $q$ larger than 7 , one can see a difference in the long time limit between the blocked (NN) and the unblocked 

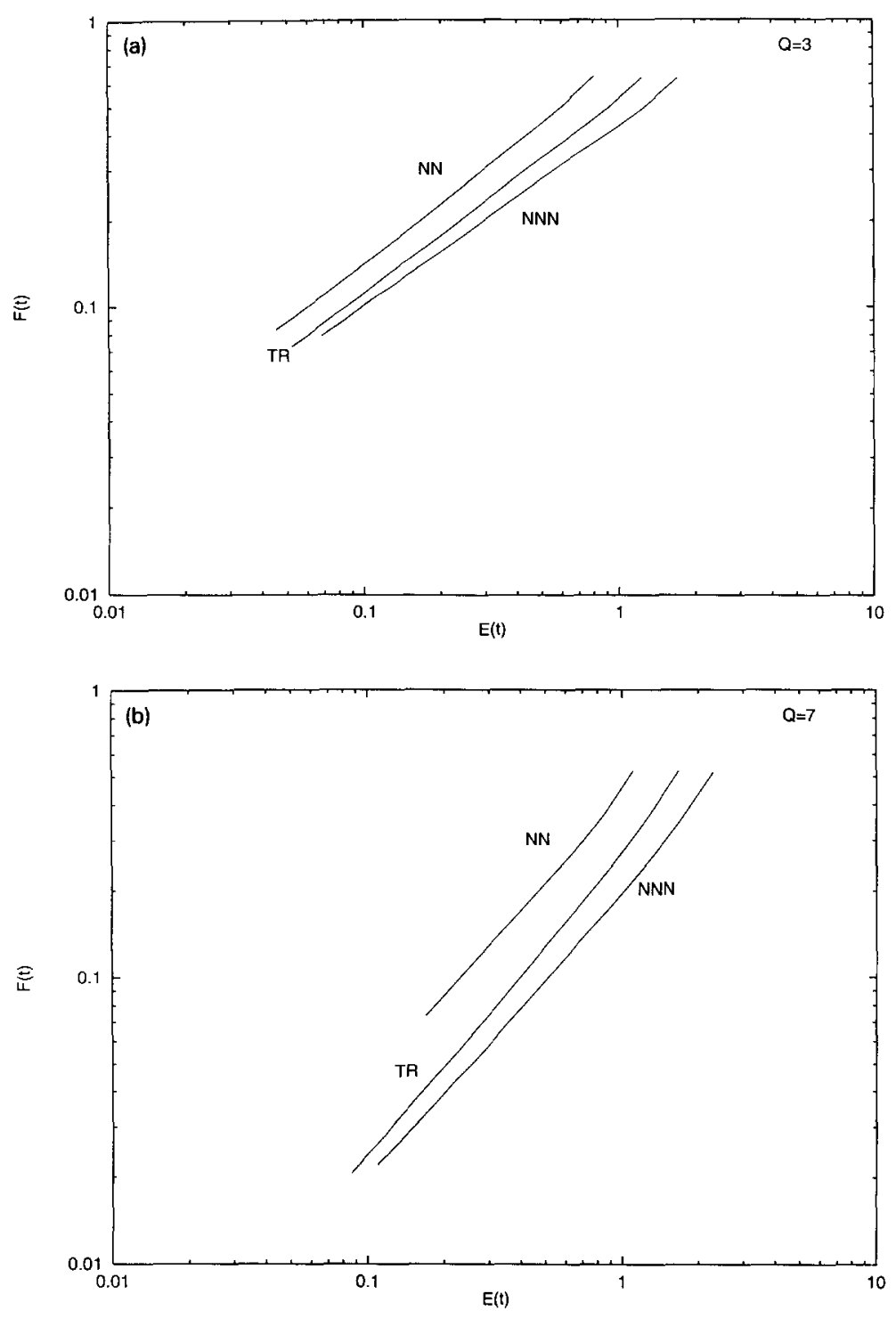

Fig. 4. $\log -\log$ plot of $F(t)$ versus $E(t)$ for the three lattices when $q=3,7, \infty$. Except for NN at infinite $q$, we do not regard the minor differences in the slopes as asymptotically significant.

(TR and NNN) cases (Fig. 4). When blocking effects are visible, ( $q>7$ on the NN lattice), the range of variation of the energy is too small to allow a reliable prediction of an exponent.

The values of $\phi$ given in Table 1 represent the main result of this work. Of course it would be interesting to develop a theory able to predict these exponents. So far we could only prove that $\phi \geq 2$ for $q=\infty$ : if the size of domains is $D(t)$, certainly $F(t) \leq D(t)^{-2}$ since (for $q=\infty$ ) there is at most one spin in each domain which never flipped since the beginning of the simulation. Then using the fact that at zero 


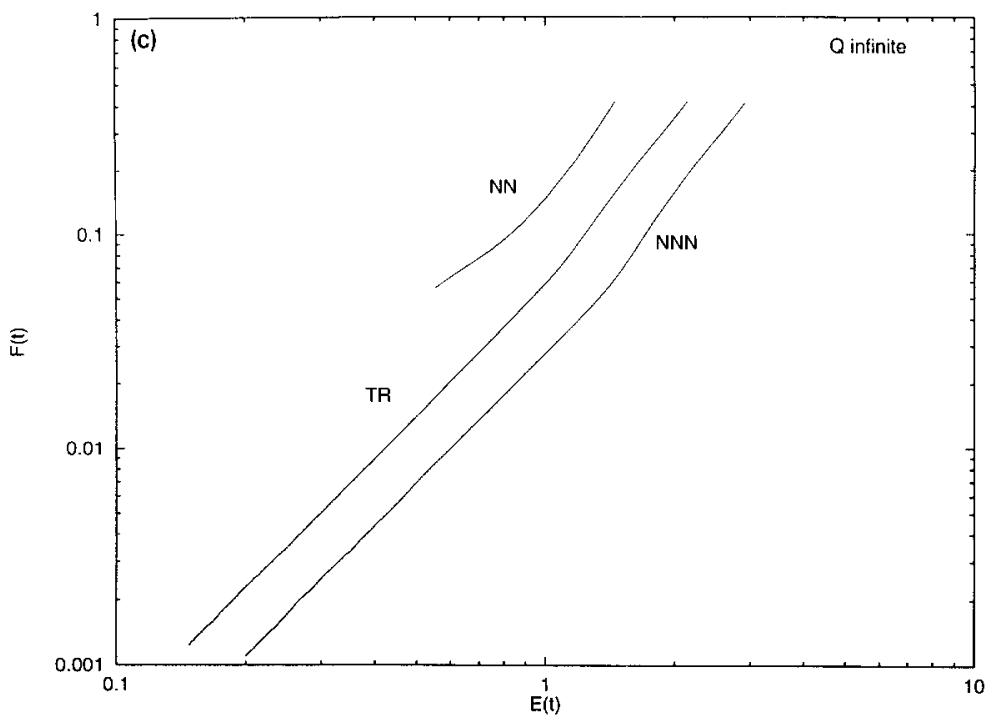

Fig. 4-continued.

temperature $D(t)^{-1} \leq E(t)$, one finds that $\phi \geq 2$. It is remarkable that 2 seems to be the right value, meaning probably that domains do not move much while they grow.

For large $q$, our data suggest that $\phi$ is different in the blocked (NN) and in the unblocked (TR and NNN) case. Certainly, the growth due to the motion of defects in blocked configurations would deserve more attention. In particular, since the growth mechanisms are completely different in the unblocked and in the blocked case, it would be interesting to know whether properties other than time dependences are sensitive to this difference.

Several approximate schemes have been discussed to describe growth processes $[1,3-$ $5,21]$. Some of them give a remarkable agreement with the results of simulations. It would be an additional test on their quality if they were able to predict $\phi$ accurately.

Lastly, it would be interesting to attack the problem at finite temperature. Obviously at finite temperature, the number of spins which never flip decreases exponentially with time. However, it is meaningful to ask what fraction of the volume remains always in the same phase (at least when the size of domains is much larger than the bulk correlation length). The main problem is to define the microscopic quantity allowing to measure that property in Monte Carlo simulations.

PMCO and DS thank H.J. Herrmann for hospitality at ESPCI, and the Brazilian agencies FAPERJ, CNPq, CAPES and FINEP for partial support of PMCO.

\section{References}

| I| A.J. Bray, Adv. Phys. 43 (1994) 357.

12] P.C. Hohenberg and B.I. Halperin, Rev. Mod. Phys. 49 ( 1977) 435.

13| T. Ohta, D. Jasnow and K. Kawasaki, Phys. Rev. Lett. 49 (1982) 1223.

14| G.F. Mazenko, Phys. Rev. B 42 ( 1990) 4487. 
|5| F. Liu and G.F. Mazenko, Phys. Rev. B 44 (1991) 9185.

|6| D.S. Fisher and D.A. Huse, Phys. Rev. B 38 (1988) 373.

17| P.S. Sahni, D.J. Srolovitz, G.S. Grest, M.P. Anderson and S.A. Safran, Phys. Rev. B 28 (1983) 2705.

|8| G.S. Grest, D.J. Srolovitz and M.P. Anderson, Phys. Rev. B 38 (1988) 4752.

19| B. Derrida, A.J. Bray and C. Godrèche, J. Phys. A 27 (1994) L357.

|10| D. Stauffer J. Phys. A 27 (1994) 5029.

111 ) S.N. Majumdar and D.A. Huse, Phys. Rev. E 52 (1995) 270.

112| H. Flyvbjerg, Phys. Rev. E 47 (1988) 4037.

|1.3| J.A. Glazier, S.P. Gross and J. Stavans, Phys. Rev. A 36 (1987) 306.

1 14| M.P. Anderson, G.S. Grest and D.J. Srolovitz and S.A. Safran, Philos. Mag. 59 (1989) 293.

|15| D. Stauffer and P.M.C. de Oliveira, Physica A 215 (1995) 407.

$|16|$ U. Gropengiesser, Physica $A$, in press.

|17| B. Derrida, V. Hakim and V. Pasquier, Phys. Rev. Lett. 75 (1995) 751.

| $18 \mid$ B. Derrida, J. Phys. A 28 (1995) 1481.

[19] F.Y. Wu, Rev. Mod. Phys. 54 (1982) 235.

$120 \mid$ M. Lau, C. Dasgupta and O.T. Valls, Phys. Rev. B 38 (1988) 9024.

$121 \mid$ C. Sire and S.N. Majumdar, Phys. Rev. E 52 (1995) 244.

|22| J. Adler, Physica A 171 (1991) 453.

|23| P.M.C. de Oliveira, Computing Boolean Statistical Models (World Scientific, Singapore, 1991). 